DESY 04-230

\title{
The spectrum of $\mathrm{SU}(N)$ gauge theories in finite volume
}

\author{
Harvey B. Meyer ${ }^{1}$ \\ Deutsches Elektronen-Synchrotron DESY \\ Platanenallee 6 \\ D-15738 Zeuthen \\ harvey.meyer@desy.de
}

\begin{abstract}
We compute the spatial-volume dependence of the spectrum of $4 \mathrm{D} \mathrm{SU}(3 \leq N \leq 6)$ gauge theories by lattice Monte-Carlo techniques. Setting the scale with the string tension, the spatial volume is $L^{3}$ with $0.78 \mathrm{fm} \leq L \leq 2.3 \mathrm{fm}$. The Euclidean 'time' direction is kept large enough to be considered infinite and the boundary conditions are periodic in all four dimensions. We study the mixing of torelon pairs with the scalar and tensor glueballs, using a $2 \times 2$ Hamiltonian based on large- $N$ counting rules. Looking to the other symmetry channels, finite-volume effects on the glueball spectrum are already surprisingly small in SU(3), and they become rapidly smaller as $N$ is increased: several low-lying $\mathrm{SU}(6)$ states have no finite-volume corrections at the 1-2\% level, at least down to $L=0.9 \mathrm{fm}$. We discuss the relation of this work with analytic calculations in small and intermediate volume, and with Eguchi-Kawai reduction in the planar limit.
\end{abstract}

\footnotetext{
${ }^{1}$ On leave from: Rudolf Peierls Centre for Theoretical Physics, University of Oxford, Oxford OX1 3NP, U.K.
} 


\section{Introduction}

It has long been realised that the volume dependence of $\mathrm{SU}(N)$ gauge theories is an important source of information on their dynamics. In very small spatial volumes $L \Lambda \ll 1$, perturbation theory is applicable due to asymptotic freedom and dimensional reduction effectively occurs with respect to all three space dimensions (we assume periodic boundary conditions). This idea was used in [1] to derive an effective Hamiltonian for the zero modes of the pure gauge theory, which allows one to compute the 'glueball' spectrum analytically. The technique was then gradually extended by taking into account tunneling between perturbative vacua to reach volumes corresponding roughly to $0.5 \mathrm{fm}$ [2] (for a review, see [3]). After pioneering smallvolume Monte-Carlo calculations by Berg and Billoire [4, the analytic results were found to be in agreement with numerical data for the $\mathrm{SU}(2)$ gauge group in the much-improved calculations [5] 6] of the late eighties; numerical results for the $\mathrm{SU}(3)$ gauge group remained more limited [7. Also in the presence of light quarks, finite-volume techniques have proven useful to gain insight into the theory. An effective small-volume action was derived in [6] for the $\mathrm{SU}(2)$ gauge theory including the one-loop contribution of light quarks, and later extended to intermediate volumes [8]. The existence of analytic results in small volume, even at finite lattice spacing 9], was exploited in [10] to study the scaling behaviour of various lattice gauge actions. More recently, the so-called $\epsilon$-regime, defined by $m_{\pi} L \ll 1$, has been used as a testing ground for chiral perturbation theory, through the connexion with random matrix theory [11.

At intermediate volumes, $L \simeq 1 \mathrm{fm}$, non-perturbative effects are an obstacle for purely analytic calculations. However, physical quantities of the finite-volume theory can be related to observables of the corresponding infinite-volume theory. The finite-volume correction to the mass of a stable state is proportional to $e^{-\frac{\sqrt{3}}{2} \bar{m} L}$ (where $\bar{m}$ is the mass gap) and the proportionality constant is nothing but the forward scattering amplitude of two of the lightest particles in infinite volume [12, 13. Further, unstable states will mix with the scattering states of their decay products in a finite volume, and the mass splittings inform about the phase shifts in infinite volume, and in particular on the width of the resonance [14, 15]. Similarly matrix elements in finite volume can be related to the 'weak' decay rate of 'kaons' in infinite volume [16.

Another point of view on gauge theories in finite-volume is obtained from t'Hooft's large$N$ expansion, where $N$ is the number of colours [17. Indeed, large- $N$ QCD is thought to be a theory of free mesons and glueballs (while baryons have masses of order $N$ [18]); this picture is supported by arguments involving the large- $N$ counting rules for Feynman diagrams and the assumption of confinement at $N=\infty$. From the general relations between finite and infinite observables cited above, one immediately infers that finite-volume effects should become weaker as $N$ increases, since widths and scattering amplitudes are suppressed by a power of $1 / N$. Conversely, while the low-lying $\mathrm{SU}(N)$ glueball spectrum in large volume has been shown to have $1 / N^{2}$ corrections [33, 34, the finite-volume effects on energy levels can be used to determine non-perturbatively the $N$-dependence of certain three- and four-point functions. This work can be seen as a first attempt in this direction. By comparison, measuring these $n$-point functions on the lattice in a straightforward way would be extremely hard.

The argument on the size of finite-volume effects outlined above fits in with the ideas of reduction initiated by Eguchi and Kawai [19]: the $\mathrm{SU}(\infty)$ lattice gauge theory defined on a single lattice hypercube of size $a^{4}$ has equivalent loop equations for its Wilson lines to those of the corresponding theory on an infinite lattice, and corrections come from the connected correlation of traced Wilson loops, which is suppressed by $1 / N^{2}$. In fact the proof given 
by the authors trivially extends to any $\hat{L}^{4}$ lattice, $L=\hat{L} a$. Thus if the vacua of the theory, defined (a) on the $\hat{L}^{4}$ lattice and (b) on the infinite lattice, 'choose' the same class of solutions, finite-volume effects on Wilson loop expectation values vanish at $N=\infty$.

The equivalence of the loop equations holds provided that the centre symmetry $Z(N)$ (which is promoted to a global $U(1)$ symmetry at $N=\infty$ ) does not break spontaneously. Strictly speaking, at finite $N$ such a spontaneous symmetry breaking cannot occur in finite volume. But the energy barrier between the different vacua allowed by the symmetry grows with $N$ so that tunnelling between them becomes completely suppressed at $N=\infty$. This point can be made more precise. In the Euclidean path integral formulation one may reinterpret one of the 'small' spatial dimensions as the Euclidean 'time' direction, which fixes the temperature, $T=1 / L>T_{c}$. In this picture, two of the three spatial dimensions have size $L$ and one is infinite (or at least $\geq L$ ). The thermal tunnelling from one vacuum to the other was discussed in 20] in a semi-classical way. In this process two different vacua must coexist 'temporarily', and a domain wall must appear between the confined and one of the $N$ deconfined vacua. Once the 'temperature' $1 / L$ is large, perturbation theory becomes applicable. The domain wall tension between two $Z(N)$ vacua is given by [21]

$$
\sigma_{w}\left(T=\frac{1}{L}\right)=\frac{b_{k}(N, L)}{L^{3}}, \quad b_{k}=k(N-k) \frac{4 \pi^{2}}{3 \sqrt{3 \lambda(1 / L)}} ; \quad \lambda=g^{2} N .
$$

If a domain wall orthogonal to the large dimension separates two $k$-vacua, its energy is $\sigma_{w} L^{2}$. At temperature $T=\frac{1}{L}$, the tunnelling rate is thus proportional to the Boltzmann factor $e^{-b_{k}(N, L)}$. We note that due to asymptotic freedom, even at fixed $N$ the Boltzmann factor is suppressed as $L \rightarrow 0$, albeit more slowly than a power law. To obtain the tunnelling rate this expression ought to be multiplied by further factors that take into account the entropy of domain walls; however they are not expected to depend strongly on $N$, and therefore we conclude that the tunnelling is exponentially suppressed in $N$. At large $N$ the exponent is large, and this justifies the semi-classical method and language used in the argument. If we now return to the original interpretation of the system as a finite-volume one at zero temperature, we see that from the point of view of Monte-Carlo simulations, the centre symmetry along one of the dimensions of size $L$ is practically broken permanently at finite, large $N$. This conclusion does not depend on the size of the dimension left unspecified, in particular it holds for $L^{4}$ and $L^{3} \times \infty$ lattices.

It is well-known that if one takes $\hat{L}=1$ the spontaneous symmetry breaking does occur (in the sense outlined above) with periodic boundary conditions, a fact that led to the 'quenched' 22] and 'twisted' 23] Eguchi-Kawai models. In the former case, the path integral is constrained in a way that maintains a uniform distribution of eigenvalues of the link matrices on the unit circle. In the latter case, the symmetry breaking is prevented by twisted boundary conditions [27. In fact, the connexion between the twisted reduced model and the ordinary gauge field theory is then even more precise: for the choice of symmetric twist, the reduced theory at finite- $N$ has loop equations equivalent (up to the $O\left(1 / N^{2}\right)$ corrections to factorisation) to those of the corresponding theory on a periodic $\hat{L}^{4}$ lattice, with $\hat{L}_{N}=\sqrt{N}$. In particular, at large but fixed $N,\left\langle\operatorname{Tr} U_{\mu}\right\rangle=0$ and $\left\langle\left(\operatorname{Tr} U_{\mu}\right)^{\hat{L}_{N}}\right\rangle=0$, but the latter (a Polyakov loop in the field theory) will have a very long autocorrelation time at sufficiently weak coupling. Therefore, as one takes the continuum limit, $N$ has to be increased so as to maintain the physical size of the box $L=a \hat{L}_{N}$ in which the gauge theory is defined in order to converge to the expected theory. 
To compare the spectrum of the (partially) reduced and the original theory, connected twopoint functions must be considered. A Hamiltonian version of the reduction (on an $L^{3} \times \mathbf{R}$ lattice) was discussed in [26] and arguments for the equality of the spectra in the reduced model and in the infinite-volume theory were given. In essence, one of the arguments is that the reduction property still holds if a source term for closed Wilson loops is added to the action; since this is a generating functional for glueball correlation functions, the finitevolume corrections to the spectrum must also vanish in the planar limit. For the argument to be complete, however, the equality of the generating functionals must be shown to hold beyond the linear term in the source.

Factorisation implies that the $N=\infty$ theory corresponds to a classical theory. In the coherent state method [24, the spectrum could in principle be obtained by expanding the classical Hamiltonian (including the $O\left(1 / N^{2}\right)$ corrections) to quadratic order around the point of phase space corresponding to the vacuum state.

In this work we investigate by Monte-Carlo techniques the finite-spatial-volume spectrum of the $\mathrm{SU}(3), \mathrm{SU}(4)$ and $\mathrm{SU}(6)$ gauge theories, for $0.78 \mathrm{fm} \leq L \leq 2 \mathrm{fm}$. By keeping $L>1 / T_{c}$, we will avoid that the system gets locked into a $Z(N)$ broken phase for long periods of MonteCarlo time (especially for $S U(4)$ and $S U(6)$ ). We study separately the symmetry sectors where 'additional' states occur and those where there are only 'intrinsic' finite-volume effects on the states already present in the infinite-volume theory. In particular we examine the $N$ dependence of the finite-volume effects in both cases.

In the next section we describe in more detail the theoretical expectations on the behaviour of the finite-volume spectrum of pure gauge theories. We present the lattice results for the $\mathrm{SU}(3)$ gauge group (section 3), and then $\mathrm{SU}(4)$ and $\mathrm{SU}(6)$ (section 4). We finish with a summary of the results and a general discussion.

\section{Finite-volume and planar physics}

We consider a four-dimensional Euclidean quantum field theory defined on a spatial hypertorus with an infinite time direction, $V=L^{3} \times \mathbf{R}$. In a quantum field theory admitting a mass gap $\bar{m}$, the leading finite-volume effect on the spectrum normally corresponds to the emission, propagation 'around the world' and reabsorption of the lightest particle. A formula corresponding to this picture was established to all orders in perturbation theory in [12]: the

mass shifts behave as $e^{-\frac{\sqrt{3}}{2} \bar{m} L}$, and the formula is accurate when the exponent is large. A simple picture for the origin of this mass shift is that the particle interacts via a Yukawa potential with its 'replica' located by periodicity in $L \times L \times L$ cells. It is now tempting to jump to the conclusion that the spectrum only has exponentially small corrections. However the situation changes qualitatively if a 'new' light state appears at volumes where $L$ is still large compared to the inverse mass gap of the infinite-volume theory - a non-perturbative effect. This is precisely the situation of pure $\mathrm{SU}(N)$ gauge theories, where such an extra state is present in a finite box but becomes a very massive and broad resonance which decouples in the infinite-volume limit. We now review in some detail how this happens.

Due to the global $Z(N)$ symmetry in the $\mathrm{SU}(N)$ gauge theory, the eigenstates of the transfer matrix can be classified according to their winding number. Let us encode the $Z(N)$ transformation properties of an operator or state by a triplet $\left(e_{x}, e_{y}, e_{z}\right)$ [13. A glueball has $(0,0,0)$, a torelon in the $\hat{x}$ direction has $(1,0,0)$, the triplets combine additively for a direct product of states and the entries are defined modulo $N$; moreover, charge conjugation connects 
the sector $k$ to $N-k$. In particular, it turns out that the lightest state for $L<1.5 \mathrm{fm}$ is the torelon, which acquires the phase $e^{i 2 \pi / N}$ under a centre transformation. One can then imagine a state made of two torelons winding the same cycle of the hypertorus in opposite directions. Such an object is invariant under the $Z(N)$ symmetry, and therefore becomes part of the glueball spectrum. The three resulting states (one for each spatial direction) can then be classified into the singlet $A_{1}^{++}$and the doublet $E^{++}$of the cubic irreducible representations. In general there is a splitting between these two 'extra glueball states'.

Apart from the simple torelon pairs, one could imagine that even more states appear in the $(0,0,0)$ sector in small volumes. For $\mathrm{SU}(N), N$ torelons winding a cycle of the torus in the same direction transform trivially under the centre of the gauge group. The real part of such operators couple to the $A_{1}++$ and $E^{++}$sectors, while the imaginary part belongs to the $T_{1}^{--}$triplet. For $k \leq N / 2$, two $(k, 0,0)$ operators constitute another possibility to construct glueball operators. One can also consider operators transforming non-trivially under two or three of the $Z(N)$ symmetries, $(1,1,0)$ and $(1,1,1)$; two of them, winding in opposite direction, again couple to $(0,0,0)$ states. However, in the absence of interactions these states are all significantly heavier than the lightest two states in the representations we shall consider. In the following we shall see no numerical evidence that they play a role in the dynamics of the low-lying spectrum.

At $L \simeq 0.8 \mathrm{fm}$, the single-torelon mass is significantly lighter than half the lightest scalar glueball mass in large volume. Therefore, in the absence of interactions, the lightest state in the $A_{1}^{++}$(and $E^{++}$) channels would be a two-torelon scattering state. The interaction potential between the two zero-momentum states is in general suppressed by their dilution in the transverse directions, $\Delta V \propto\left(\ell_{\perp} / L\right)^{2}$ in our case, where $\ell_{\perp}$ is the average width of the 'string'; but this is hardly a suppression at the volumes we are interested in. Also, (working in the basis of states with definite relative momentum) the higher relative momenta $\frac{2 \pi n}{L}$ will contribute little to the ground state for $L<1 \mathrm{fm}$.

Since the torelon mass varies roughly proportionally to $L$ and the glueball mass is only weakly dependent on $L$, we expect the dynamics of torelons and their mixing with an ordinary glueball state to determine the mass of the two lightest $A_{1}^{++}$(and $E^{++}$) states in a certain range of volumes. It turns out that it corresponds roughly to $0.8 \mathrm{fm} \leq L \leq 1.2 \mathrm{fm}$. In that range, there is a large energy gap to the third state in these two representations. That means that to a good (and controllable) approximation, we can study the system with a $2 \times 2$ effective Hamiltonian.

In order to apply the stationary perturbation theory of quantum mechanics, we need to choose a starting orthonormal basis which diagonalises an 'unperturbed' Hamiltonian. We suppose here that the full Hamiltonian of the $\mathrm{SU}(N)$ gauge theory can be expanded in inverse powers of $1 / N$ :

$$
H(L, N)=\sum_{k=0}^{\infty} \frac{H_{k}(L)}{N^{k}} .
$$

The existence of the t'Hooft limit implies that $H_{o}$ has the same eigenvalues as the Hamiltonian of the $\mathrm{SU}(\infty)$ theory $^{2}$; the latter, according to conventional wisdom, describes non-interacting colour singlets. $H_{o}(L)$ is our 'unperturbed' Hamiltonian. If $m_{T}(L, N)$ is the torelon mass and $m_{G}(L, N)$ the glueball mass in the relevant symmetry channel, then the restriction of $H_{o}(L)$

\footnotetext{
${ }^{2}$ The $S U(\infty)$ theory may have additional, decoupled sectors of free hadrons.
} 
to the vector space span by these two states reads

$$
H_{o}(L)=\left(\begin{array}{cc}
2 m_{T}(L, \infty) & 0 \\
0 & m_{G}(L, \infty)
\end{array}\right) .
$$

We now consider the full hamiltonian $H(L, N)$ in the same basis:

$$
H_{2 \times 2}(L, N)=\left(\begin{array}{cc}
E_{2 T}(L, N) & h(L, N) \\
h(L, N) & m_{G}(L, N)
\end{array}\right)
$$

with

$$
E_{2 T}(L, N)=2 m_{T}(L, \infty)+v_{T}(L, N), \quad m_{G}(L, N)=m_{G}(L, \infty)+v_{G}(L, N) .
$$

The diagonal matrix elements $v_{T}$ and $v_{G}$ contain the terms describing 'intrinsic' finite-volume corrections of the unperturbed states. For instance, in an effective low-energy description, the force between flux-tubes is attractive as a result of the exchange of the lightest particle, a scalar glueball; such a force is always attractive. The glueball state also undergoes 'intrinsic' corrections, proportional in leading approximation to $e^{-c \bar{m} L}$ [1]. However, equally important are the off-diagonal elements describing mixing. Pictorially one can imagine the following process: two flux-tubes undergo 'fusion', after which they form a closed, contractible flux loop. In the flux-tube model [29], a glueball precisely corresponds to such a loop: at this point the uncertainty principle (and possibly curvature effects) prevent the loop from shrinking to a point.

Let us now discuss the $N$ dependence of the various contributions to the Hamiltonian (4). The finite- $N$ energy-shift $v_{G}$ corresponds to the usual $1 / N^{2}$ correction to the mass of a coloursinglet state, along with the virtual emission 'around the world' and reabsorption of a coloursinglet by a colour-singlet state, which is also a $1 / N^{2}$ effect. Similarly $v_{T}(L)$ contains $O\left(1 / N^{2}\right)$ corrections to the mass of each torelon, and the 'elastic' interaction of torelons encoded in $v_{T}(L)$ proceeds through the exchange of a colour-singlet, and therefore is also $O\left(1 / N^{2}\right) . h(L)$ on the other hand, corresponds to the decay amplitude of a colour-singlet into two coloursinglets, which is $O(1 / N)$. A simple way to see this is that the decay rate of a glueball above the two-torelon threshold (if the transverse dimensions were large) would be proportional to $h^{2}$ in this formalism, according to 'Fermi's golden rule'. On the other hand, the width of colour-singlets in the pure gauge theory is $O\left(1 / N^{2}\right)$, which leads to the announced conclusion.

Thus we expect, at sufficiently large $N$, the functions $v_{G}, v_{T}$ and $h$ to take the functional forms

$$
\begin{aligned}
v_{G}(L, N) & \sim \frac{1}{N^{2}} \bar{v}_{G}(L) \\
v_{T}(L, N) & \sim \frac{1}{N^{2}} \bar{v}_{T}(L) \\
h(L, N) & \sim \frac{1}{N} \bar{h}(L) .
\end{aligned}
$$

On the lattice, we of course compute what corresponds to the two eigenvalues of $H_{2 \times 2}(L, N)$, $m_{o}(L, N)$ and $m_{1}(L, N)$. Suppressing everywhere the $(L, N)$ dependence, one has

$$
\begin{aligned}
m_{o}+m_{1} & =E_{2 T}+m_{G} \\
\left(m_{o}-m_{1}\right)^{2} & =\left(E_{2 T}-m_{G}\right)^{2}+4 h^{2}
\end{aligned}
$$


The information extracted from the lattice is in general not sufficient to determine all three unknown functions $v_{G}, v_{T}$ and $h$. However the large- $N$ counting rules can help us out.

Firstly, according to the latter,

$$
m_{T}(L, N)=m_{T}(L, \infty)+O\left(1 / N^{2}\right) .
$$

For the glueball mass, similarly

$$
m_{G}(L=\infty, N)=m_{G}(L=\infty, N=\infty)+O\left(1 / N^{2}\right),
$$

since in large volume there is no other light state the glueball can mix with, and

$$
m_{G}(L=\infty, N=\infty)=m_{G}(L, N=\infty)
$$

if the planar theory has no finite-volume corrections. Therefore, using Eqs. [5, 6, 17, 11, 12, and 13. Eq. 9 becomes the prediction

$$
m_{o}(L, N)+m_{1}(L, N)=2 m_{T}(L, N)+m_{G}(L=\infty, N)+O\left(1 / N^{2}\right)
$$

that can easily be tested on the lattice, since all quantities are at a given $N$.

Secondly, the half-splitting of the two lattice states provides an upper bound for $h(L, N)$ :

$$
|h(L, N)| \leq \frac{1}{2}\left|m_{o}(L, N)-m_{1}(L, N)\right| \text {. }
$$

For $L$ such that

$$
1-\frac{E_{2 T}(L, N)}{m_{G}(L, N)} \ll O(1 / N),
$$

Eq. 10 tells us that $h(L, N)$ saturates the upper-bound, up to small corrections. Since $E_{2 T}(L, N)$ and $m_{G}(L, N)$ are not known a priori, it is more convenient to express the condition above by trading them for the known quantities $2 m_{T}(L, N)$ and $m_{G}(L=\infty, N)$ :

$$
1-\frac{2 m_{T}(L, N)}{m_{G}(\infty, N)} \ll O(1 / N),
$$

(the left-hand side of 16 and 17 only differ by $O\left(1 / N^{2}\right)$ terms). If $h(L, N)$ is found to be $O(1 / N)$, the procedure used to extract $h$ is self-consistent. What is interesting, from the point of view of $1 / N$ counting, is that in the volumes satisfying Eqn. 17 the finite-volume mass shift is of order $1 / N$. Because in large volume glueballs are relatively heavy in units of the string tension, it is easy to see that this condition will necessarily be satisfied at some intermediate box size. Thus there are circumstances where the expectation that finite-volume mass shifts are suppressed by $1 / N^{2}$ can be wrong. Analogous violations of the ' $1 / N^{2}$ correction rule' for the spectrum of $\mathrm{SU}(N)$ gauge theory will be discussed in section 5 .

In conclusion our expectations are the following. In most representations of the cubic group, where no degeneracies appear as the volume is varied, the energy levels have small deviations from their infinite-volume values. These corrections are of order $1 / N^{2}$ and behave as $e^{-c \bar{m}(L) L}$ (as long as the exponent is large). In those representations where degeneracies appear, such as the $A_{1}^{++}$and $E^{++}$which contain torelon pairs, an interesting interplay between the parameters $L$ and $N$ appears. When $L$ is such that the relative separation of states is much larger than $O(1 / N)$, the deviations of the glueball masses from their infinite-volume values are $O\left(1 / N^{2}\right)$. But as the torelon mass comes within $O(1 / N)$ of half the infinite-volume glueball mass, the finite-volume corrections to glueball masses are enhanced through mixing to $O(1 / N)$. In parallel, as the torelon-pair energy falls below the lightest $A_{1}^{++}$glueball, the mass gap $\bar{m}(L)$ is given by that energy and finite-volume shifts become more rapid, as a function of $L$. 


\section{Lattice results for $\mathrm{SU}(3)$}

Our lattice calculations employ the standard plaquette action and the equally standard 1:4 combination of heatbath and over-relaxation sweeps is used for the update. We calculate ground and excited state masses $m$ in the zero-flux sector $(0,0,0)$ from Euclidean correlation functions of zero-momentum operators using standard variational techniques [31. We extract the string tension, $\sigma$, from the mass of a flux loop that closes around a spatial torus. We perform calculations for a fixed value of the inverse bare coupling $\beta=6 / \mathrm{g}^{2}=6.0$ corresponding to a lattice spacing $a \simeq 0.097 \mathrm{fm}$, if we use $\sqrt{\sigma}=440 \mathrm{MeV}$. The calculations are on lattices ranging from $8^{3} 48$ to $24^{3} 24$, corresponding to a spatial extent $0.78 \mathrm{fm} \leq L \leq 2.3 \mathrm{fm}$. Since some states become very light on small volumes, the time direction is gradually increased from 24 to 48 lattice spacings as the spatial volume is reduced in order to suppress 'around the world' contributions (proportional to $e^{-m\left(L_{t}-t\right)}$ ) to the correlation function $C(t)$. In the $\hat{L}=8$ case we checked that increasing $\hat{L}_{t}$ to 64 does not alter the measured spectrum.

It is well-known that it is crucial to include sufficiently different operators in the variational basis that overlap significantly onto the low-lying spectrum, particularly when one is trying to extract more than one energy level. From the discussion of the previous section, it should be clear that what is required in the $A_{1}^{++}$and $E^{++}$sectors are contractible Wilson loops as well as pairs of spatial Polyakov loops winding around a cycle of the spatial torus in opposite directions. We measure them with zero relative momentum, since a crude estimate of the energy of two non-interacting torelons with one unit of momentum $2 \pi / L$ each turns out to be well above the lightest $A_{1}^{++}$state for the $L$ of interest ${ }^{3}$. We take the linear combinations of the scattering states that belong to the $A_{1}^{++}$and $E^{++}$representations of $O_{h}$. We note that the overlaps between the two-torelon and the Wilson loop operators is substantial in the region $L \simeq 1 \mathrm{fm}$ (see below). In the other representations, our basis of operators consists only of appropriate linear combinations of contractible Wilson loops. All our operators, the Wilson loops and the scattering state of Polyakov loops, are constructed with several levels of smearing and blocking (see e.g. [30]). We note however that one must be careful in a small volume not to include operators so non-local that they contain paths winding around a cycle of the torus.

In some of the simulations we use a 2-level algorithm [32] that reduces the variance of rapidly decaying correlators, thereby allowing us to extract the mass at larger time-separation. This is especially relevant in small volumes, where it turns out that our fuzzy operators do not necessarily have as good overlaps on the physical states as in large volumes. On the smallest volume we notice a significant increase in the auto-correlation time of the spatial Polyakov loop expectation value (see Fig. 2 both of these runs were employing the ordinary algorithm). This may be attributed to the proximity of $L$ to $1 / T_{c}$. $a T_{c} \simeq 7.25$ according to [20], and it appears that tunnelling transitions are moderately frequent on the $8^{3} \times 48$ lattice. In such a situation a possible criterion to split up the data into independent blocks is to require that the magnitude of the average Polyakov loop in each block be less than a prescribed value. Our jackknife bins cover a Monte-Carlo time that is larger than the width of a typical peak on Fig. 2. The way we store the measurements [32] in the 2-level algorithm allows us to study in detail the dependence of the statistical errors on the jackknife bins; we are confident that our statistical errors are not biased by more than $10 \%$.

Table 1 contains our data on the various cubic representations. In the next section we

\footnotetext{
${ }^{3}$ We reserve the name 'adjoint Polyakov loop' for the case where the Polyakov loops are created at the same position. The use of such operators was pioneered by Berg and Billoire [4].
} 
study the lightest few states in the $A_{1}^{++}$('scalar') and $E^{++}$('tensor') channels before we move on to the other representations.

\section{Channels with the lightest states}

The evolution of the lightest $A_{1}^{++}$and $E^{++}$states is illustrated on Fig. 3. Although we are only really interested in the first two states, we monitor the level of the third excited state to estimate the mass gap to the next states. We also plot the $T_{2}^{++}$states which are degenerate with the $E^{++}$states in the large volume (and continuum) limit.

We observe that below $1.2 \mathrm{fm}$, there are two states under $2 \mathrm{GeV}$, rather than one; they are the $A_{1}^{++}$and $A_{1}^{++*}$ states. Meanwhile the $A_{1}^{++* *}$ is approximately degenerate with the large-voume $A_{1}^{++*}$.

The straightforward interpretation, namely that the fundamental state becomes lighter, and that the $n^{\text {th }}$ excited state becomes degenerate with the large-volume $(n-1)^{\text {th }}$ excited state $(n=1,2, \ldots)$, would be hard to understand in a simple way. A far more natural interpretation is the one developed in the previous section, namely that one particular state, associated with the torelon pair, becomes light on the small volumes. It crosses the level of the large-volume $A_{1}^{++*}$ state around $L=1.5 \mathrm{fm}$, and that of the large-volume $A_{1}^{++}$level around $L=1 \mathrm{fm}$. Each level-crossing is accompanied by a maximal mixing of the torelon pair with the glueball state.

A fit to the lightest energy level for $\hat{L} \geq 12$ was attempted, assuming the functional form to $m(L)=m_{\infty}-\frac{C}{L} \exp \left(-\frac{\sqrt{3}}{2} m_{\infty} L\right)$ [12]. The fit is acceptable but we find that the factor $C$ is large $(670 \pm 150)$ and driven by the point at $\hat{L}=12$, signalling that the glueball exchange 'around-the-world' is probably not the dominant effect in that range of volumes (such a large factor was already found in [35]). The two lightest states come closest together at $L=1 \mathrm{fm}$. In a two-state mixing model, the identity of the two states at smaller $L$ is inverted: the ordinary glueball component of the first-excited state is larger than that of the ground state, which is now more of the torelon-pair nature. This crossing apparently happens when the curve $m_{o}(L)$ has an inflexion point. This interpretation is supported by the fact that our two-torelon operators have significant overlaps ${ }^{4}$ with the usual scalar glueball operators $(0.32$ at $\hat{L}=11$, 0.67 at $\hat{L}=10$ and 0.55 at $\hat{L}=9$, choosing each time our best operator of each type).

A similar pattern is seen in the $E^{++}$channel: the ground state is pushed down by the presence of an extra light state for $L \leq 1.2 \mathrm{fm}$. Below that $L$, the first excited state is roughly degenerate with the fundamental $T_{2}^{++}$state, which has only small finite-volume corrections. The same mixing and crossing interpretation thus seems appropriate: on the smaller volumes it is the first-excited state which has a glueball-dominated wave function.

\section{Two-state mixing model}

At $\hat{L}=12$, we observe a state with an energy of almost exactly $2 m_{T}(L)$, both in the $A_{1}^{++}$ and $E^{++}$sectors, and the other light state still has very small finite-volume corrections with respect to the large-volume glueball masses. However the situation changes quite rapidly for $\hat{L} \leq 11$. Compared to other symmetry channels, the scalar and tensor glueballs masses suddenly undergo finite-size shifts larger by about a factor of 2 .

The near-degeneracy of the two lightest states in the region $0.9-1.1 \mathrm{fm}$ suggests the application of the two-state mixing model. Fig. 1 shows the average mass of the fundamental and first excited states (separately in the $A_{1}^{++}$and $E^{++}$sectors). The lattice data is compared

\footnotetext{
${ }^{4}$ The overlap is defined as $\frac{\left\langle O_{1} \mid O_{2}\right\rangle}{\sqrt{\left\langle O_{1} \mid O_{1}\right\rangle\left\langle O_{2} \mid O_{2}\right\rangle}}$.
} 
to the energy level $m_{T}(L)+m_{G}(\infty) / 2$ that one expects if torelon interactions and intrinsic finite-volume corrections to the glueball mass vanish (see Eq. 14). The agreement is good for $L \geq 0.9 \mathrm{fm}$, while at smaller $L$ the true average mass is pushed up compared to the naive estimate. This provides evidence that the 'intrinsic' effects are small in the region of interest, though one could be fooled if the mass shift for the torelon-pair has opposite sign to that of the finite-volume glueball mass, which would result in a large cancellation of these effects in the quantity considered.

Ignoring this possibility for the moment, if we now suppose (in the spirit of large- $N$ counting rules) that the 'unperturbed' energy levels are separately well described by the values $2 m_{T}(L)$ and $m_{G}(\infty)$, we can estimate the mixing strength $h(L)$ through Eqn. 10. For $\hat{L}=10$ we find $h(L)=h \simeq 0.15(2) m_{G}(\infty)$ (the quoted error is statistical). In the $E^{++}$sector, the splitting is smaller than $\left|m_{G}(\infty)-2 m_{T}(L)\right|$ in the $0.9-1.1 \mathrm{fm}$ region, and mixing can only split the states further apart. Perhaps unsurprisingly we must conclude that $N=3$ is not yet large enough for the $N$-counting rules of section 2 to be reliable in the extraction of the mixing strength $h$. Nevertheless the mass splitting, given in Table 4 provides an upper bound on $h(L)$.

\section{Other representations}

In the other representations the complications discussed above do not occur. We consider the $T_{2}^{++}, E^{-+}, T_{2}^{-+}, A^{-+}$and $T_{1}^{+-}$representations (see Figs. 3] 4). The $T_{2}^{++}$state is the easiest case, because of its relatively small mass. As mentioned above, its volume dependence is weak and smooth. The same holds for the $E^{-+}, T_{2}^{-+}$and $T_{1}^{+-}$states, whose masses increase monotonically as the volume becomes smaller. The increase is at the $10 \%$ level at $L \simeq 0.88 \mathrm{fm}$, as we discuss more quantitatively below. Unfortunately our calculation in the 'pseudoscalar' channel $A_{1}^{-+}$is not sufficiently good to allow us to make precise statements about its volume dependence, but it is clear that it is the only one of these states that is becoming lighter - its mass seems to fall off rather abruptly around $1 \mathrm{fm}$. We remark that our operators had poor overlap onto the state on the smaller volumes, whilst having satisfactory overlap on the large volumes.

\section{Larger $N$}

We repeat the calculation of the spectrum for $\mathrm{SU}(4)$ and $\mathrm{SU}(6)$; our method is entirely analogous to the $\mathrm{SU}(3)$ case. The lattice spacing is slightly larger, $a=0.11 \mathrm{fm}$ if we set the scale with the string tension, but prior knowledge 33 indicates that the system is already in the scaling region at that lattice spacing. In the $A_{1}^{++}$and $E^{++}$sectors, we again include the two-torelon operators along with the Wilson loops. Zero-momentum scattering states of two loops with $\mathcal{N}$-ality $k=2$ were not included, although they could have a substantial overlap with one of the first excited scalar glueballs for $N \geq 4$, if its wavefunction were well described by a closed $k=2$ string. This possibility has recently been suggested [34, but we leave it as

a subject of investigation for the future. The $\mathrm{SU}(4)$ and $\mathrm{SU}(6)$ spectra are given in Table 2 and 3 .

\section{$N$-dependence of 'intrinsic' finite-volume effects}

We start with the simpler case, namely the comparison of states not affected by the torelon 
pairs. On Fig. [5 (top) we compare the relative finite-volume mass shifts

$$
\Delta(L) \equiv \frac{m(L)}{m\left(L_{\infty}\right)}-1
$$

of the lightest state between $L \simeq 0.85 \mathrm{fm}$ and $L=1.2 \mathrm{fm}$ for the representations $T_{2}^{++}, T_{1}^{+-}$, $E^{-+}$and $T_{2}^{-+}$( $L_{\infty}$ corresponds to our largest lattice). Note that the error bars on $m(L)$ and $m(L=\infty)$ are independent and therefore combine in quadrature. We already observed in the previous section that the variations were small in $\mathrm{SU}(3)$, at the $10 \%$ level for $L \simeq 0.88 \mathrm{fm}$. Here we see that the finite-volume dependence gets even more suppressed as one moves from $\mathrm{SU}(3)$ to $\mathrm{SU}(4)$ and $\mathrm{SU}(6)$. This suppression is rapid: for $N=6$ our data is consistent with vanishing finite-volume effects, in spite of relatively small error bars $\left(1-2 \%\right.$ for the $T_{2}^{++}$and $T_{1}^{+-}$cases). Thus determining the $N$ functional dependence numerically will require very accurate data. On the other hand the finite-volume corrections vary quite smoothly with $L$ in these representations, and therefore it is sufficient to compare different $\mathrm{SU}(N)$ theories at one chosen value of $\sqrt{\sigma} L \simeq 2$, for instance.

\section{$N$ dependence of torelon-pair mixing with glueballs}

As we showed in the $\mathrm{SU}(3)$ case, the spectrum in the $A_{1}^{++}$and $E^{++}$sectors is affected by the presence of torelon pairs. The average mass and the mass splitting, that appear on the left-hand-side of Eqs. 9 and 10 are given in Table 4. Recall that the half-splitting provides an upper-bound for $h(L, N)$ (Eqn. 15). Our data shows that the mixing strength is modest in magnitude but the determination of its $N$ dependence requires accurate data for several $L$ in the region of maximal mixing given by Eqn. 17] Note that the minimum of the half-splitting as a function of $L$ gives $h$ only if the latter is $L$ independent.

Considering the bottom part of Fig. [5 we see that the finite-volume mass shifts, although smaller than for $\mathrm{SU}(3)$, remain significant also for $\mathrm{SU}(4)$ and $\mathrm{SU}(6)$ in these representations, and that they are strongly dependent on the precise value of $L$. Due to the crossing of states, the shift changes sign around $1 \mathrm{fm}$ (we used the state which is closest to $m_{G}(L=\infty)$ to compute the relative mass shift). These observations are consistent with the expectations on the $N$ dependence of finite-volume effects formulated in section 2 .

\section{Discussion}

We have studied the finite-size evolution of the low-lying glueball spectrum as a function of the number of colours in $\mathrm{SU}(N)$ gauge theories. The box is $L^{3}$ in size, with $0.78 \mathrm{fm} \leq L \leq 2.3 \mathrm{fm}$, the boundary conditions are periodic and the lattice spacing is fixed at roughly $0.1 \mathrm{fm}$. Seven symmetry channels were investigated. In most of them $\left(T_{2}^{++}, E^{-+}, T_{2}^{-+}, T_{1}^{+-}\right)$, the states observed connect smoothly with their infinite-volume counterparts. In the $\mathrm{SU}(3)$ case they become gradually more massive, and the mass shift is only at the $10 \%$ level at $0.8 \mathrm{fm}$. For $\mathrm{SU}(4)$ and $\mathrm{SU}(6)$ this mass shift is very suppressed, and the $\mathrm{SU}(6)$ data is in fact consistent with vanishing finite-volume effects. This constitutes the first observation of the large- $N$ suppression of these effects on the spectrum in Monte-Carlo simulations. To show that these effects are truly of order $1 / N^{2}$ will require very accurate data. We also note that asymptotically Lüscher's formula [12, 13. predicts a lowering of the glueball masses as $L$ decreases, since the exchange of the lightest scalar glueball yields an attractive force; we conclude that the effect 
we are seeing is a different one, and that the asymptotic correction is too small to be observed with the present statistical accuracy.

In the $A_{1}^{-+}$'pseudoscalar' sector, the lightest glueball becomes significantly lighter with respect to the infinite-volume state, and since this is observed for all gauge groups considered we think that this state deserves a dedicated study. One might speculate that the mass shift is related to the lower average $Q^{2}$ in small volumes ( $Q$ is the topological charge). In that case, one would also expect the pseudoscalar glueball to show up as a lighter state in the presence of light dynamical quarks, which suppress the topological charge fluctuations. We note that a surprisingly sharp onset of the validity of random matrix theory in predicting the distribution of eigenvalues of the overlap Dirac operator in different topological sectors on quenched configurations was observed around $1.1 \mathrm{fm}$ in [11. It is not excluded that the behaviour of the lightest pseudoscalar glueball is related to that observation.

The $A_{1}^{++}$(scalar) and $E^{++}$(tensor) sectors are most simply interpreted in terms of the appearance of extra light states, with respect to the large volume situation. These have a natural interpretation in terms of pairs of flux loops winding around one cycle of the hypertorus in opposite directions. Around $1 \mathrm{fm}$ the states come close to the ground states in these sectors. A simple two-state mixing model was used to interpret the two lowest energy levels. It appears that the binding energy of the torelons and the intrinsic mass shifts of the glueballs are rather small, so that the main cause of the variation of the spectrum in the $L=1 \mathrm{fm}$ region is mixing. In $\mathrm{SU}(3)$ it was estimated that the mixing energy amounts to about $15 \%$ of the infinite-volume glueball mass in the scalar sector. This estimate still has a very large systematic uncertainty; the present study of this quantity must be viewed as exploratory. However we note that in practice it is easy to tune $L$ so that Eqn. [17 holds; one can thus expect to determine the $N$ dependence of the mixing energy $h$ in the near future. Theoretical arguments were given in section 2 why the mixing energy should be of order $1 / N$ and therefore the dominant effect at large $N$ in the cross-over region.

The issue of mixing of near-degenerate states in the large $N$ expansion is an important and general one. For instance, a $k=2$ string [28] of length $L$ can be thought of as a state resulting from the mixing of two 'unperturbed' states ${ }^{5}$ which both have $1 / N^{2}$ corrections to their energy with respect to the planar limit value $2 \sigma L$ ( $\sigma$ is the fundamental string tension). However a mixing energy of order $1 / N$ implies that the lightest $k=2$ string energy deviates by a term of that order from $2 \sigma L[38$. Thus studying the mixing strength of torelon pairs with glueballs in intermediate volumes satisfying Eqn. 17] addresses the same basic issue concerning $\mathrm{SU}(N)$ gauge theories in a physical context where it is much easier for lattice calculations to give a clear-cut answer.

To extend this work to $L<1 / T_{c}$, one has to confront the problem of long auto-correlation times on any quantity sensitive to the centre symmetry $Z(N)$. This is especially true at large $N$, as outlined in the introduction. It then appears to be more advisable to use twisted boundary conditions, as was done for $\mathrm{SU}(2)$ in the work of Stephenson and Teper 36] following the analytic work [37. Working with larger gauge groups would then allow one to test the theoretical ideas of reduction. Eventually volumes are reached where perturbation theory becomes applicable and accurate $(L \simeq 0.2 \mathrm{fm})$, so that the connexion with ab initio analytic methods [1] can be established. It would thus be interesting to investigate the spectrum of the effective small-volume Hamiltonian for the $\mathrm{SU}(N)$ theory, $N$ large. A necessary and non-trivial condition to give agreement with the large-volume spectrum, that is satisfied by

\footnotetext{
${ }^{5}$ They correspond to operators $\operatorname{Tr}\left\{P^{2}\right\}$ and $(\operatorname{Tr}\{P\})^{2}$, if $P$ is the Polyakov loop.
} 
this Hamiltonian even at finite $N$, is that it becomes spherically symmetric on very small volumes [1. In view of the lessons learnt in this work and the fact that the multi-torelon operators decouple from the glueball-creating operators at large $N$, we would expect only certain energy levels to match those of the gauge theory in infinite volume.

\section{Acknowledgements}

I am indebted to all participants of an informal seminar set up by M. Teper and M. Schvellinger in Oxford, where several subjects touched upon in this article were discussed. In particular I am grateful to H. Vairinhos for his presentation of Eguchi-Kawai reduction. The numerical calculations were performed on a PPARC and EPSRC funded Beowulf cluster of the Rudolf Peierls Centre for Theoretical Physics.

\section{References}

[1] M. Luscher, Nucl. Phys. B 219 (1983) 233; M. Luscher and G. Munster, Nucl. Phys. B 232 (1984) 445.

[2] J. Koller and P. van Baal, Nucl. Phys. B 302 (1988) 1;

[3] P. van Baal, arXiv:hep-ph/0008206.

[4] B. Berg and A. Billoire, Phys. Lett. B 166 (1986) 203 [Erratum-ibid. 185B (1987) 446];

B. Berg, A. Billoire and C. Vohwinkel, Phys. Rev. Lett. 57 (1986) 400.

[5] B. A. Berg and A. H. Billoire, Phys. Rev. D 40, 550 (1989); C. Michael, G. A. Tickle and M. J. Teper, Phys. Lett. B 207 (1988) 313.

[6] J. Kripfganz and C. Michael, Phys. Lett. B 209 (1988) 77.

[7] C. Vohwinkel and B. A. Berg, Phys. Rev. D 40, 584 (1989).

[8] C. Michael, Nucl. Phys. B 329 (1990) 225.

[9] P. van Baal, Phys. Lett. B 224 (1989) 397.

[10] M. Garcia Perez, J. Snippe and P. van Baal, arXiv:hep-lat/9607007.

[11] L. Giusti, M. Luscher, P. Weisz and H. Wittig, JHEP 0311 (2003) 023 arXiv:hep-lat/0309189.

[12] M. Luscher, Commun. Math. Phys. 104 (1986) 177.

[13] M. Luscher, DESY-88-156 Lectures given at Summer School 'Fields, Strings and Critical Phenomena', Les Houches, France, Jun 28 - Aug 5, 1988

[14] M. Luscher, Commun. Math. Phys. 105 (1986) 153.

[15] M. Luscher, Nucl. Phys. B 364 (1991) 237.

[16] L. Lellouch and M. Luscher, Commun. Math. Phys. 219 (2001) 31 arXiv:hep-lat/0003023. 
[17] G. 't Hooft, Nucl. Phys. B 72, 461 (1974).

[18] E. Witten, Nucl. Phys. B 160 (1979) 57.

[19] T. Eguchi and H. Kawai, Phys. Rev. Lett. 48, 1063 (1982).

[20] B. Lucini, M. Teper and U. Wenger, JHEP 0401 (2004) 061 arXiv:hep-lat/0307017.

[21] T. Bhattacharya, A. Gocksch, C. Korthals Altes and R. D. Pisarski, Nucl. Phys. B 383, 497 (1992) arXiv:hep-ph/9205231; T. Bhattacharya, A. Gocksch, C. Korthals Altes and R. D. Pisarski, Phys. Rev. Lett. 66, 998 (1991).

[22] G. Bhanot, U. M. Heller and H. Neuberger, Phys. Lett. B 113, 47 (1982).

[23] A. Gonzalez-Arroyo and M. Okawa, Phys. Lett. B 120, 174 (1983); A. Gonzalez-Arroyo and M. Okawa, Phys. Rev. D 27, 2397 (1983).

[24] L. G. Yaffe, Rev. Mod. Phys. 54, 407 (1982).

[25] J. Kiskis, R. Narayanan and H. Neuberger, Phys. Lett. B 574 (2003) 65 arXiv:hep-lat/0308033.

[26] H. Levine and H. Neuberger, Phys. Rev. Lett. 49, 1603 (1982).

[27] G. 't Hooft, Nucl. Phys. B 153 (1979) 141.

[28] B. Lucini and M. Teper, Phys. Rev. D 64 (2001) 105019 arXiv:hep-lat/0107007.

[29] N. Isgur and J. Paton, Phys. Rev. D 31 (1985) 2910.

[30] B. Lucini, M. Teper and U. Wenger, JHEP 0406 (2004) 012 arXiv:hep-lat/0404008.

[31] C. Michael, Nucl. Phys. B 259 (1985) 58; M. Luscher and U. Wolff, Nucl. Phys. B339, 222 (1990).

[32] H. B. Meyer, JHEP 0301 (2003) 048 hep-lat/0209145; H. B. Meyer, JHEP 0401 (2004) 030 hep-lat/0312034.

[33] B. Lucini and M. Teper, JHEP 0106 (2001) 050 arXiv:hep-lat/0103027.

[34] H. B. Meyer and M. J. Teper, arXiv:hep-ph/0409183 H. B. Meyer, D.Phil. thesis, Oxford 2004.

[35] P. de Forcrand, G. Schierholz, H. Schneider and M. Teper, Phys. Lett. B 152 (1985) 107.

[36] P. W. Stephenson and M. Teper, Nucl. Phys. B 327 (1989) 307.

[37] A. Gonzalez Arroyo and C. P. Korthals Altes, Nucl. Phys. B 311 (1988) 433.

[38] C.P. Korthals Altes and H.B. Meyer, in preparation.

[39] M. Luscher and U. Wolff, Nucl. Phys. B 339 (1990) 222. 


\begin{tabular}{cccccc}
\hline \hline IR & $\hat{L}=8$ & 9 & 10 & 11 & 12 \\
\hline torelon & $0.2107(14)$ & $0.2599(30)$ & $0.3201(51)$ & $0.4016(48)$ & $0.4585(25)$ \\
$A_{1}^{++}$ & $0.54(1)$ & $0.53(1)$ & $0.55(1)$ & $0.63(1)$ & $0.661(9)$ \\
$A_{1}^{++*}$ & $0.731(8)$ & $0.77(2)$ & $0.77(2)$ & $0.88(3)$ & $0.93(2)$ \\
$A_{1}^{++* *}$ & $1.23(4)$ & $1.24(7)$ & $1.15(3)$ & $<1.37(2)$ & $1.24(5)$ \\
$E^{++}$ & $0.519(4)$ & $0.583(16)$ & $0.67(1)$ & $0.883(16)$ & $0.91(1)$ \\
$E^{++*}$ & $1.12(2)$ & $1.07(3)$ & $1.04(1)$ & $0.97(3)$ & $1.075(7)$ \\
$E^{++* *}$ & $1.39(4)$ & $1.46(9)$ & $1.42(4)$ & $1.54(12)$ & $1.46(7)$ \\
$T_{2}^{++}$ & $1.16(2)$ & $1.18(2)$ & $1.12(1)$ & $1.127(5)$ & $1.06(1)$ \\
$T_{2}^{++*}$ & $1.46(6)$ & $1.59(8)$ & $1.55(5)$ & $<1.72(2)$ & $1.56(2)$ \\
$A_{1}^{-+}$ & $1.07(3)$ & $1.04(3)$ & $1.19(3)$ & $1.20(3)$ & $1.25(5)$ \\
$E^{-+}$ & $<1.62(2)$ & $1.51(6)$ & $1.45(4)$ & $1.42(5)$ & $1.39(6)$ \\
$T_{2}^{-+}$ & $1.52(7)$ & $1.53(5)$ & $1.35(3)$ & $1.44(4)$ & $1.41(1)$ \\
$T_{1}^{+-}$ & $1.50(6)$ & $1.46(5)$ & $1.41(3)$ & $<1.48(3)$ & $1.39(3)$ \\
\hline \hline
\end{tabular}

\begin{tabular}{ccccc}
\hline \hline IR & $\hat{L}=14$ & 16 & 20 & 24 \\
\hline torelon & $0.5739(34)$ & $0.6781(33)$ & $0.8860(64)$ & $1.082(60)$ \\
$A_{1}^{++}$ & $0.68(1)$ & $0.697(4)$ & $0.701(3)$ & $0.698(2)$ \\
$A_{1}^{++*}$ & - & $1.17(2)$ & $<1.32(3)$ & $<1.23(2)$ \\
$E^{++}$ & $1.06(2)$ & $1.060(6)$ & $1.07(1)$ & $1.073(3)$ \\
$E^{++*}$ & - & $1.43(1)$ & $<1.56(4)$ & $<1.48(2)$ \\
$T_{2}^{++}$ & - & $1.070(5)$ & - & - \\
$T_{2}^{++*}$ & - & $<1.50(1)$ & - & - \\
$A_{1}^{-+}$ & - & $1.15(1)$ & - & - \\
$E^{-+}$ & - & $1.36(1)$ & - & - \\
$T_{2}^{-+}$ & - & $1.37(1)$ & - & - \\
$T_{1}^{+-}$ & $<1.42(2)$ & $1.31(2)$ & $1.31(2)$ & $1.34(2)$ \\
\hline \hline
\end{tabular}

Table 1: The SU(3) spectrum (in lattice units) in finite volume from Monte-Carlo simulations at $\beta=6.0\left(a \simeq 0.097 \mathrm{fm}, V=L^{3} \times L_{t}\right)$. $\hat{L}_{t}$ is 24 for $\hat{L} \geq 12,32$ for $\hat{L}=9$ and 11 , and 48 for $L=8$ and 10. The method is described at the beginning of section 3 , 


\begin{tabular}{cccc}
\hline \hline IR & $\hat{L}=8$ & 10 & 16 \\
\hline torelon & $0.2555(45)$ & $0.4514(40)$ & $0.8610(53)$ \\
$A_{1}^{++}$ & $0.4859(69)$ & $0.6670(87)$ & $0.729(10)$ \\
$A_{1}^{++*}$ & $0.7297(97)$ & $0.981(12)$ & $1.338(13)$ \\
$E^{++}$ & $0.5465(71)$ & $0.893(14)$ & $1.139(11)$ \\
$E^{++*}$ & $1.1342(78)$ & $1.163(11)$ & $1.606(16)$ \\
$T_{2}^{++}$ & $1.1682(68)$ & $1.152(10)$ & $1.143(16)$ \\
$T_{2}^{++*}$ & $1.370(30)$ & $1.599(22)$ & $1.622(16)$ \\
$A_{1}^{-+}$ & $0.924(14)$ & $1.060(72)$ & $0.991(86)$ \\
$E^{-+}$ & $1.528(18)$ & $1.503(18)$ & $1.507(50)$ \\
$T_{2}^{-+}$ & $1.525(37)$ & $1.477(40)$ & $1.423(41)$ \\
$T_{1}^{+-}$ & $1.491(20)$ & $1.439(21)$ & $1.453(15)$ \\
\hline \hline
\end{tabular}

Table 2: The spatial volume dependence of SU(4) glueball masses in lattice units at $\beta=10.90$ $\left(a \simeq 0.11 \mathrm{fm}, V=L^{3} \times L_{t}\right)$. $\hat{L}_{t}=24$ for $\hat{L}=16, \hat{L}_{t}=36$ for $\hat{L}=8$ and 10 .

\begin{tabular}{ccccc}
\hline \hline IR & $\hat{L}=8$ & 10 & 16 & 20 \\
\hline torelon & $0.3096(38)$ & $0.5016(63)$ & $0.930(10)$ & $1.2101(85)$ \\
$A_{1}^{++}$ & $0.494(10)$ & $0.707(11)$ & $0.742(17)$ & $0.737(18)$ \\
$A_{1}^{++*}$ & $0.782(11)$ & $1.063(24)$ & $1.343(24)$ & $1.315(33)$ \\
$E^{++}$ & $0.589(12)$ & $0.942(27)$ & $1.153(18)$ & $1.180(12)$ \\
$E^{++*}$ & $1.160(14)$ & $1.175(17)$ & - & - \\
$T_{2}^{++}$ & $1.1786(88)$ & $1.177(12)$ & $1.167(13)$ & $1.160(10)$ \\
$A_{1}^{-+}$ & $0.941(55)$ & $1.203(26)$ & $\leq 1.127(51)$ & $\leq 1.293(19)$ \\
$E^{-+}$ & $1.532(68)$ & $\leq 1.573(28)$ & $1.498(21)$ & $\leq 1.61(2)$ \\
$T_{2}^{-+}$ & $1.532(18)$ & $1.496(26)$ & $1.498(26)$ & $1.521(16)$ \\
$T_{1}^{+-}$ & $1.487(25)$ & $1.462(27)$ & $1.474(17)$ & $1.47(2)$ \\
\hline \hline
\end{tabular}

Table 3: The spatial volume dependence of SU(6) glueball masses in lattice units at $\beta=25.05$ $\left(a \simeq 0.11 \mathrm{fm}, V=L^{3} \times L_{t}\right)$. $\hat{L}_{t}=24$ for $\hat{L}=16$ and $20, \hat{L}_{t}=36$ for $\hat{L}=8$ and 10 . 


\begin{tabular}{|c|c|c|c|c|c|c|}
\hline & IR & $\hat{L}=8$ & 9 & 10 & 11 & 12 \\
\hline \multirow{4}{*}{$\mathrm{SU}(3)$} & $A_{1}^{++}: m_{+}$ & $0.6394(44)$ & $0.648(18)$ & $0.66(2)$ & $0.760(17)$ & $0.792(13)$ \\
\hline & $m_{-}$ & $0.1708(80)$ & $0.248(18)$ & $0.22(2)$ & $0.255(30)$ & $0.279(21)$ \\
\hline & $E^{++}: m_{+}$ & $0.793(21)$ & $0.794(37)$ & $0.855(14)$ & $0.918(35)$ & $0.9935(53)$ \\
\hline & $m_{-}$ & $0.6182(93)$ & $0.478(28)$ & $0.37(1)$ & $0.120(20)$ & $0.177(10)$ \\
\hline \multirow{4}{*}{$\mathrm{SU}(4)$} & $\bar{A}_{1}^{++}: m_{+}$ & $0.601(11)$ & & $0.8239(80)$ & & \\
\hline & $m_{-}$ & $0.237(8)$ & & $0.314(13)$ & & \\
\hline & $E^{++}: m_{+}$ & $0.8415(76)$ & & $1.0268(75)$ & & \\
\hline & $m_{-}$ & $0.575(15)$ & & $0.263(12)$ & & \\
\hline \multirow{4}{*}{$\mathrm{SU}(6)$} & $\bar{A}_{1}^{++}: m_{+}$ & $0.6379(81)$ & & $0.885(14)$ & & \\
\hline & $m_{-}$ & $0.285(12)$ & & $0.355(24)$ & & \\
\hline & $E^{++}: m_{+}$ & $0.8749(79)$ & & $1.062(20)$ & & \\
\hline & $m_{-}$ & $0.582(28)$ & & $0.241(36)$ & & \\
\hline
\end{tabular}

Table 4: The averages masses $m_{+}=\left(m_{0}+m_{1}\right) / 2$ of and mass gaps $m_{-}=m_{1}-m_{o}$ between the fundamental and first-excited states in the $A_{1}^{++}$and $E^{++}$sectors (in lattice units). Statistical correlations are taken into account in the standard way by the jackknife method for $m_{+}$; for $m_{-}$, we give the mass plateau of $C_{1}(t) / C_{o}(t)$, where $C_{o}(t), C_{1}(t)$ are the correlators of respectively the fundamental and first-excited operators obtained from the variational method [39]. Its variance is again determined by the jackknife method.

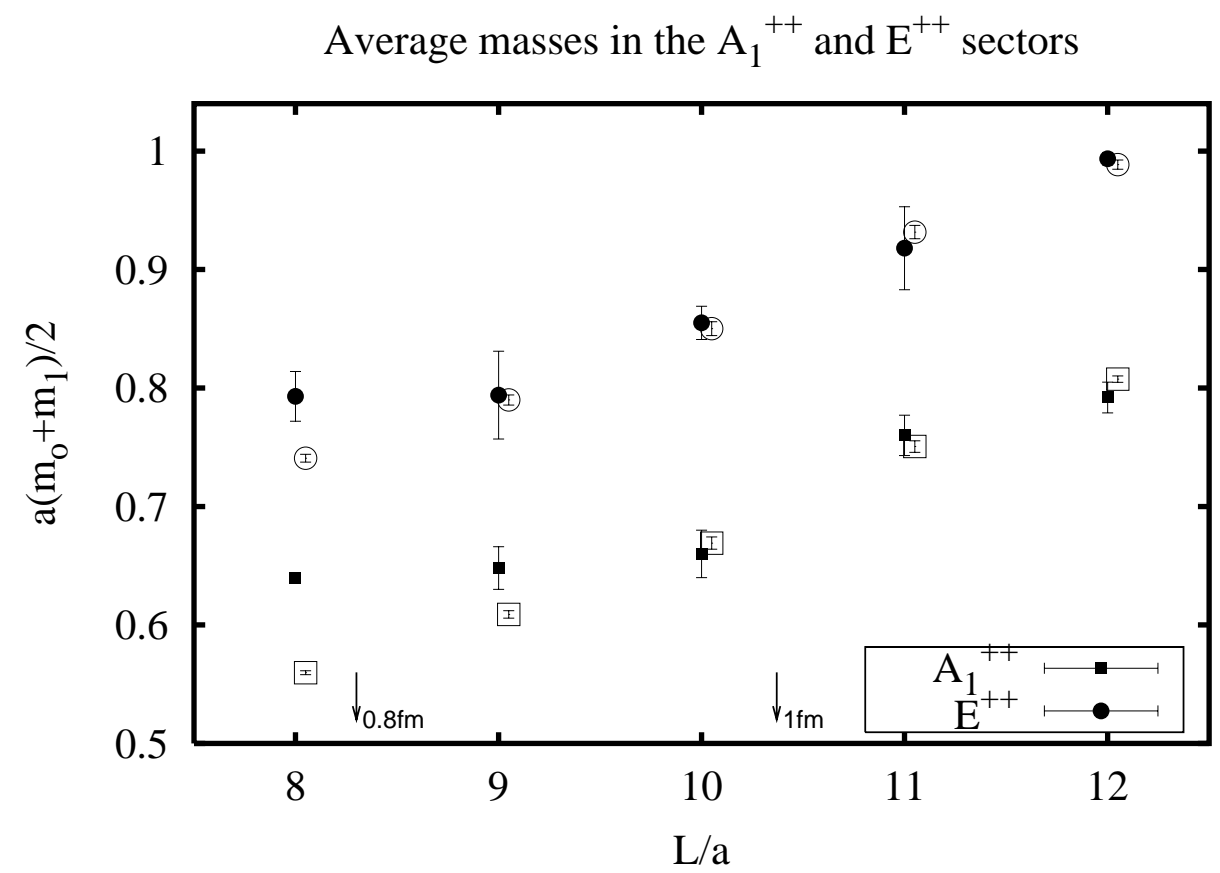

Figure 1: Average of the fundamental and first excited states in the $A_{1}^{++}$and $E^{++}$sectors (black symbols). This energy level is independent of two-state mixing. Also plotted (white symbols) is $m_{T}(L)+m_{G}(L=\infty)$. Where black and white symbol overlap, 'intrinsic' finitevolume effects are small. 


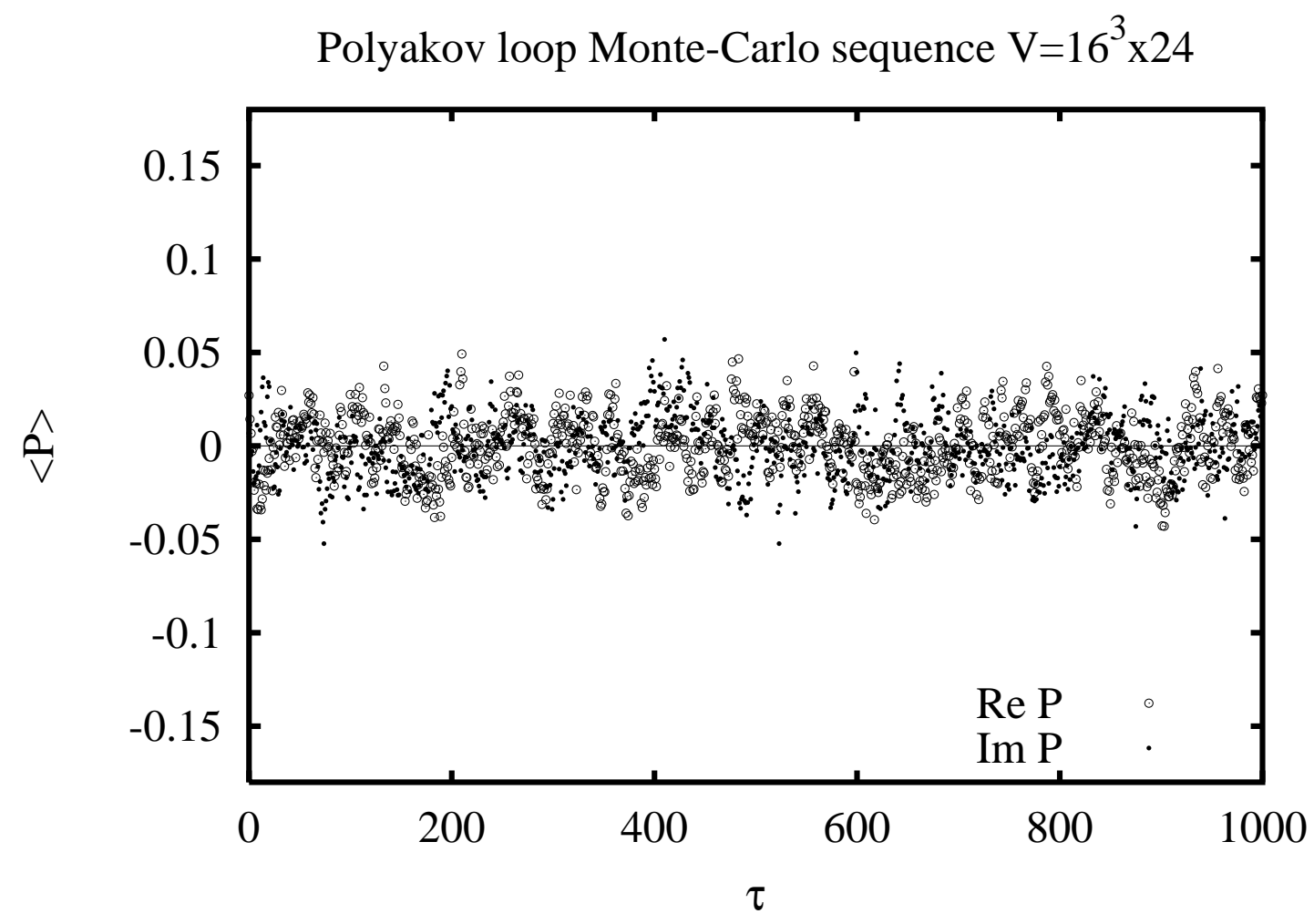

Polyakov loop Monte-Carlo sequence $V=8^{3}$ x 48

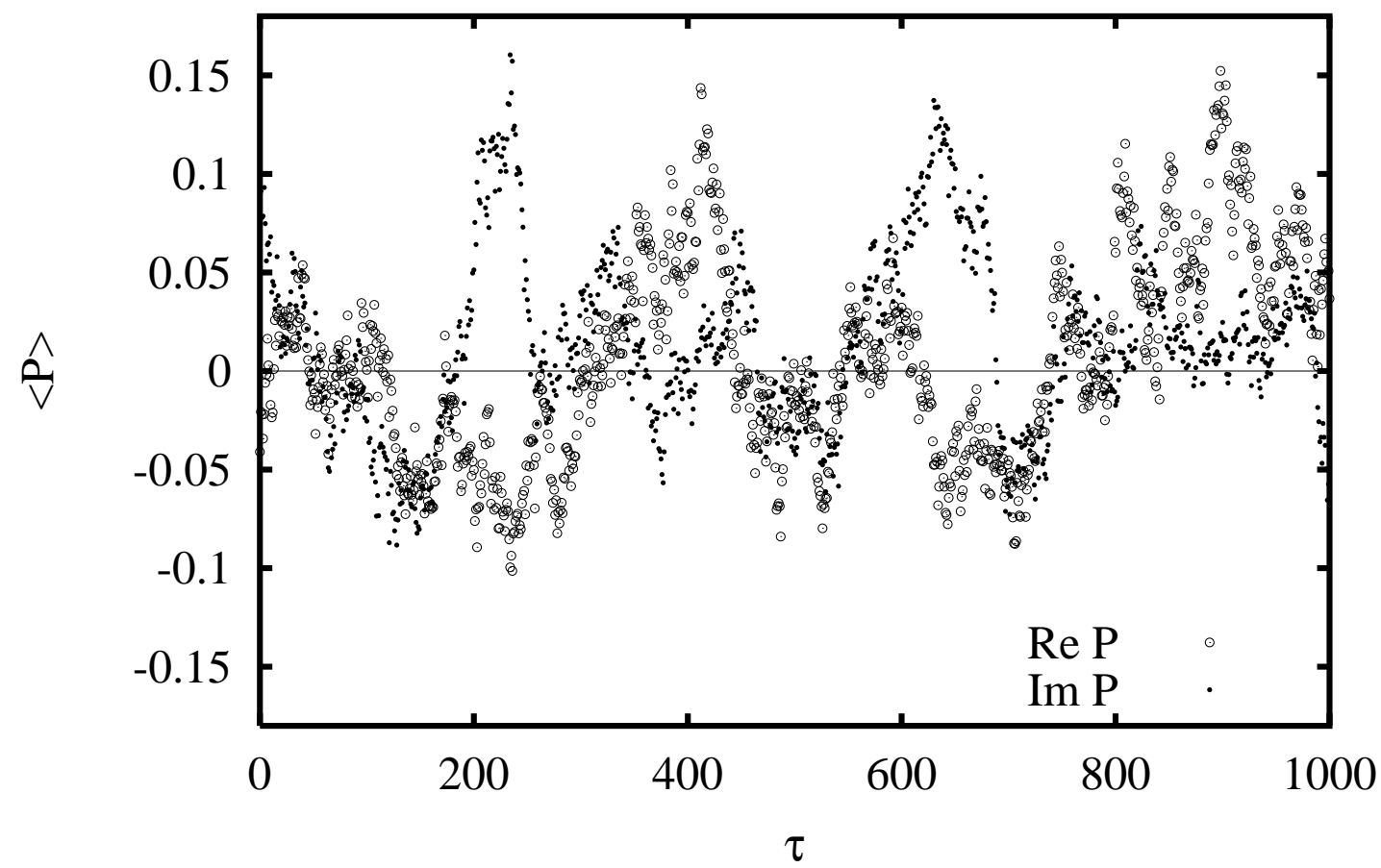

Figure 2: Monte-Carlo sequence of the average value of the spatial-torelon operators over $10^{3}$ measurements; the measurements are separated by 4 sweeps. 

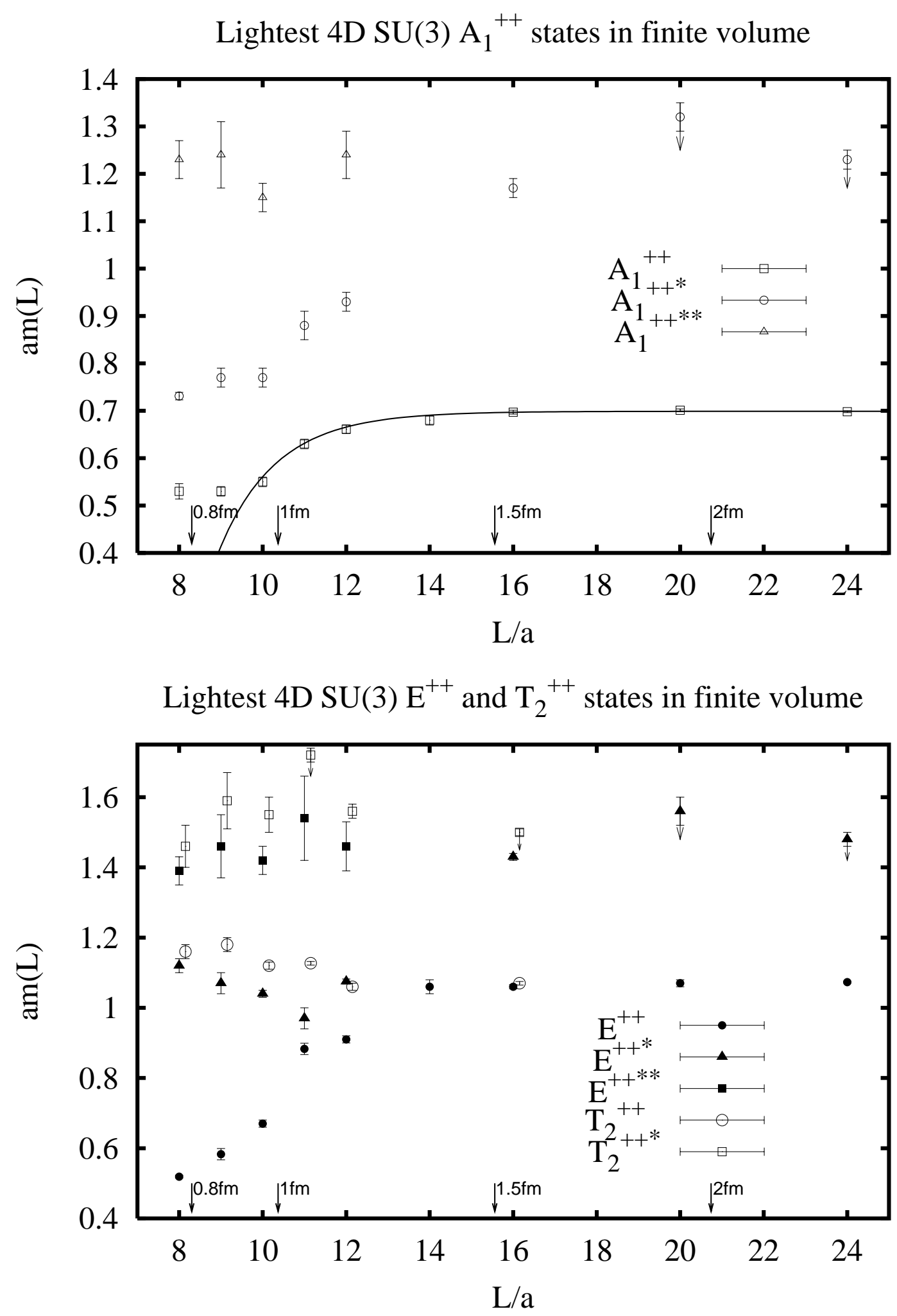

Figure 3: Volume dependence of the lightest states in the representations that mix with torelon pairs. In the top plot, the smooth curve is a fit $(0.699-674 \exp (-0.605 L) / L)$ discussed in the text. 

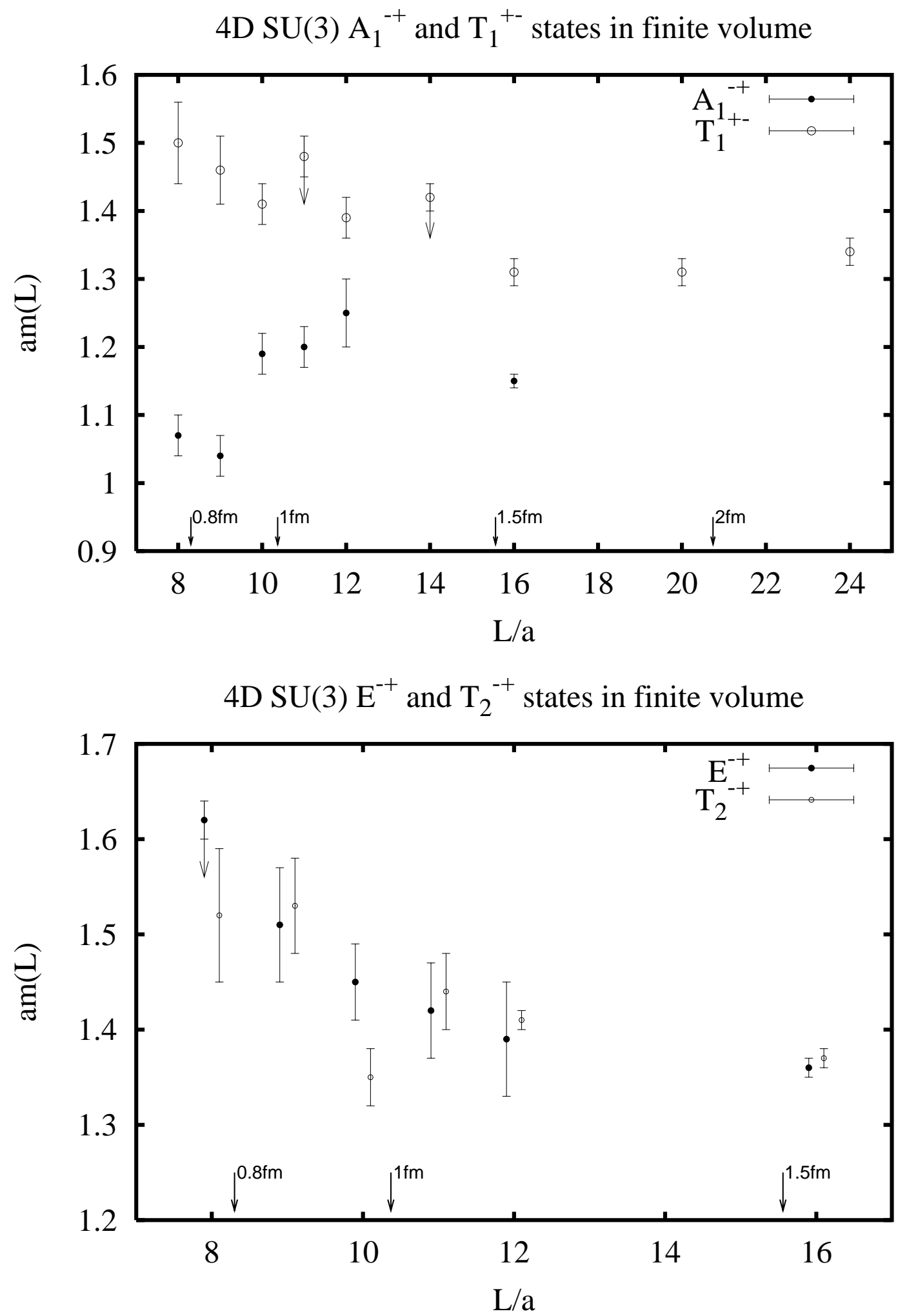

Figure 4: Volume dependence of some light states in various representations. 

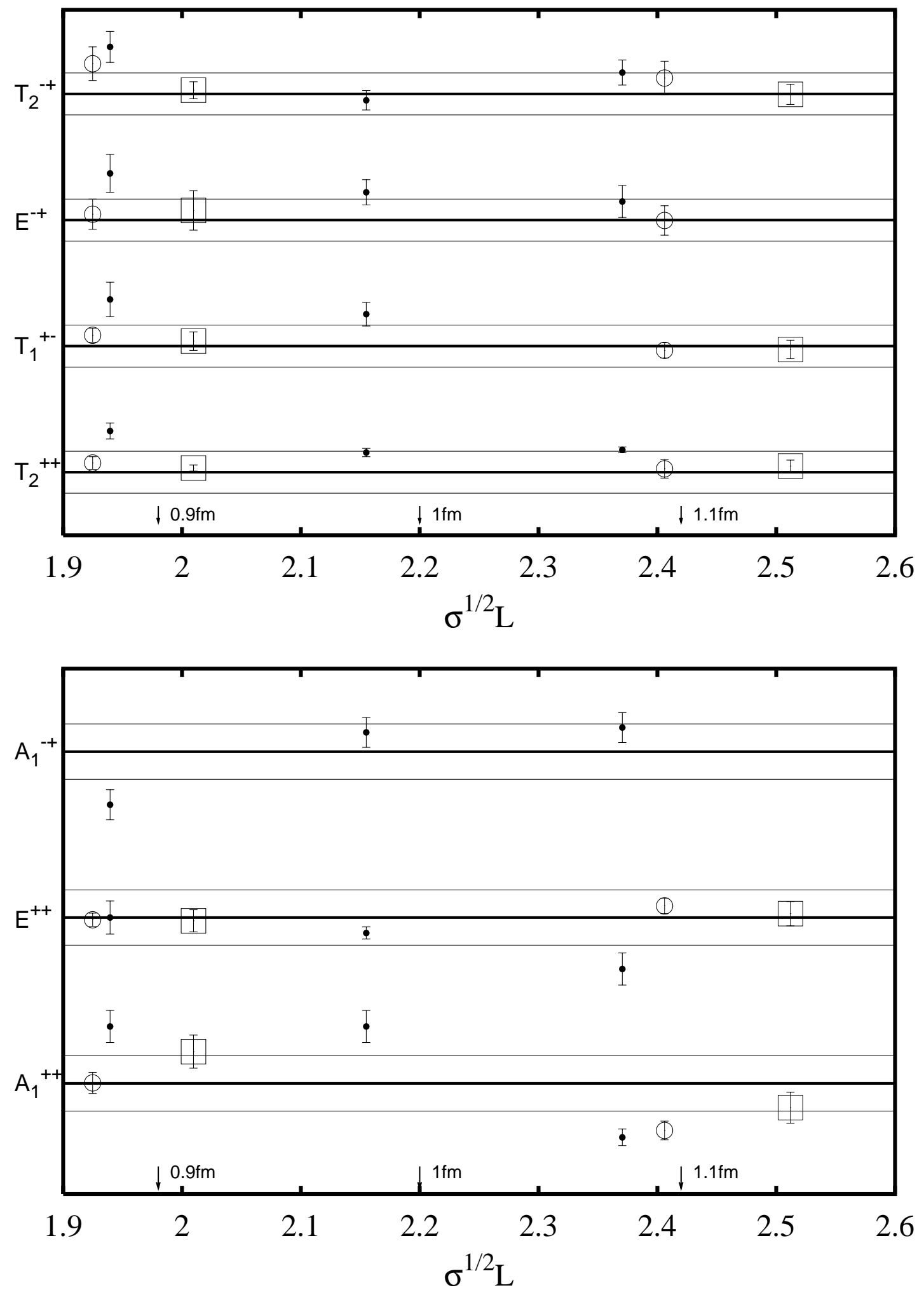

Figure 5: Finite-volume mass shifts $m(L) / m(L=\infty)-1$ in various representations; $\mathrm{SU}(3) \rightarrow$ black circles, $\mathrm{SU}(4) \rightarrow$ white circles, $\mathrm{SU}(6) \rightarrow$ white squares. The thin lines indicate the $\pm 5 \%$ levels of variation. 